\title{
Derivation of the Quantum Vlasov Equation and Dispersion Relation of a Relativistic Electron Gas
}

\author{
D. BisKamp \\ Max-Planck-Institut für Physik und Astrophysik, München \\ (Z. Naturforschg. 22 a, 869-872 [1967] ; received 25 February 1967)
}

\begin{abstract}
The selfconsistent field equation (Vhasov equation) is derived for the one-particle Wigner function of a relativistic electron-positron gas. From the linearized form we obtain the dispersion relation for any quasi-equilibrium state, which for the special case of thermal equilibrium has already been derived by Tsytovich ${ }^{1}$.
\end{abstract}

Recently some interest has arisen in the investigation of relativistic quantum plasmas. Such conditions, where temperature or density are high enough that relativistic quantum effects such as pair production become important, may exist in the interior of some far evolved or very massive stars. There exist already a number of papers that treat the relativistic electron-positron gas in thermal equilibrium especially in the strongly degenerate case which is practically the most important one ${ }^{1-3}$. It seems nevertheless to be of some inherent interest to study the relativistic electron gas out of equilibrium. For such cases the Wigner function method, which has been successfully applied for the treatment of nonrelativistic nonequilibrium plasmas ${ }^{4}$, appears to be rather convenient. Using this method BALESCU $^{5}$ recently made some first advances toward a general treatment of quantum statistical mechanics of relativistic charged particles. Such a program is certainly very hard to carry out because in addition to the problems arising from the many-particle aspect one is sure to encounter all the difficulties of ordinary quantum electrodynamics. In this note we only want to rederive the dispersion relation of a relativistic electron gas already derived and discussed for thermal equilibrium by Tsytovich ${ }^{1}$ (and in particular for $T=0$ by $\mathrm{J}_{\text {AnCovicI }}{ }^{2}$ ). This alternative derivation is more general as it applies to all "quasiequilibrium" distributions (which are possible if one neglects collisions), and thus allows an investigation of their stability.

1 V. N. Tsytovich, Soviet Phys.-JETP 13, 1249 [1961].

2 B. Jancovici, Nuovo Cim. 25, 428 [1962].

3 T. Hamada and Y. Nakamura, Progr. Theor. Phys. 36, 465 [1966].

\section{Selfconsistent Field Equation for the One-Particle Wigner Function}

We consider an electron-positron gas enclosed in a volume $\Omega$ and neutralized by a positive ion background, such that $N_{-}-N_{+}=N=$ const, $N_{-}, N_{+}$being respectively the number of electrons and positrons. Then the Hamiltonian becomes (we use the SCHRöDInger picture throughout) ${ }^{6}$ :

$$
\begin{aligned}
H= & \left.\sum_{\boldsymbol{p}}: \psi^{+}(\boldsymbol{p})(\boldsymbol{\alpha} \cdot \boldsymbol{p}+\beta m) \psi(\boldsymbol{p}):+\sum_{\boldsymbol{k}, \lambda} k a_{\boldsymbol{k} \lambda}^{+} a_{\boldsymbol{k}}\right\rangle \\
& -e \sum_{\boldsymbol{p}, \boldsymbol{k}}: \psi^{+}(\boldsymbol{p}+\boldsymbol{k}) \alpha^{\mu} \psi(\boldsymbol{p}): A_{\mu}(\boldsymbol{k})+H_{i a}, \\
\psi^{\alpha}(\boldsymbol{x})= & \frac{1}{\Omega^{1 / 2}} \sum_{\boldsymbol{p}} \psi^{\alpha}(\boldsymbol{p}) \exp \{i \boldsymbol{p} \cdot \boldsymbol{x}\}, \\
A_{\mu}(\boldsymbol{x})= & \sum_{\boldsymbol{k}} A_{\mu}(\boldsymbol{k}) \exp \{i \boldsymbol{k} \cdot \boldsymbol{x}\} \\
= & \sum_{\boldsymbol{k}, \lambda} \sqrt{\frac{2 \pi}{\Omega}} e_{\mu}^{(\lambda)}\left(\boldsymbol{a}_{\boldsymbol{k} \lambda}+\boldsymbol{a}_{-\boldsymbol{k} \lambda}^{+}\right) \exp \{i \boldsymbol{k} \cdot \boldsymbol{x}\}, \quad(1)
\end{aligned}
$$

$\alpha^{\mu}=\gamma^{0} \gamma^{\mu}=(1, \boldsymbol{\alpha})$, metric tensor $g^{\mu \mu}=(+---)$. The sign $: \ldots$ : indicates the normal product in the usual sense of field theory. The term $H_{i a}$ in (1) represents the interaction of the positive background with the electromagnetic field, which will play no part in the following considerations.

$\psi(\boldsymbol{p})$ contains the destruction operator of an electron and creation operator of a positron,

$$
\psi(\boldsymbol{p})=\psi^{(-)}(\boldsymbol{p})+\psi^{(+)}(\boldsymbol{p})
$$

[originally defined in the interaction picture as positive and negative frequency parts of $\psi(\boldsymbol{p})]$, which obey the well known commutation relations

$$
\left\{\psi^{(\mp) \alpha}(\boldsymbol{p}), \psi^{(\mp) \beta^{+}}\left(\boldsymbol{p}^{\prime}\right)\right\}_{+}=\delta_{\boldsymbol{P} \boldsymbol{p}^{\prime}} \Lambda^{\mp \alpha \beta}(\boldsymbol{p}),
$$

4 Vide e. g. W. R. Chappell and W. E. Brittin, Phys. Rev. 146, 75 [1966].

5 R. Balescu, Physica 31, 1599 [1965].

6 We use units $\hbar=c=1$. 
where $A^{\mp}(\boldsymbol{p})$ are respectively the projection operators on positive and negative free particle energy states:

$\Lambda^{\mp}(\boldsymbol{p})=\frac{E_{\boldsymbol{P}} \pm(\boldsymbol{\alpha} \cdot \boldsymbol{p}+\beta m)}{2 E_{\boldsymbol{P}}}, \quad E_{\boldsymbol{P}}=\left(\boldsymbol{p}^{2}+m^{2}\right)^{1 / 2}$.

The commutation relations of $a_{\boldsymbol{k} \lambda}, \boldsymbol{a}_{\boldsymbol{k} \lambda}^{+}$are the usual

$$
\left[a_{k \lambda}, a_{\boldsymbol{k}^{\prime} \lambda^{\prime}}^{+}\right]_{-}=\delta_{\boldsymbol{k} \boldsymbol{k}^{\prime}} \delta_{\lambda \lambda^{\prime}} \text {. }
$$

As a generalisation of the nonrelativistic concept ${ }^{4}$ relativistic WIGNER functions are to be defined as statistical averages over normal products of the field operators to provide for their vanishing in the vacuum state (no particles and photons present). In particular the one-particle Wigner function becomes:

$$
\begin{gathered}
f^{\alpha \beta}(\boldsymbol{p}, \boldsymbol{x}, t)=\frac{1}{(2 \pi)^{3}} \sum_{\boldsymbol{k}} \operatorname{Tr}\left\{\varrho(t): \psi^{+\beta}(\boldsymbol{p}+\boldsymbol{k} / 2)\right. \\
\left.\psi^{\alpha}(\boldsymbol{p}-\boldsymbol{k} / 2):\right\} \exp \{i \boldsymbol{k} \cdot \boldsymbol{x}\}
\end{gathered}
$$

with the normalisation

$$
\int_{\Omega} \mathrm{d}^{3} x \int \mathrm{d}^{3} p \operatorname{Sp}\{f(\boldsymbol{p}, \boldsymbol{x}, t)\}=N
$$

$(\operatorname{Tr}\{\varrho \ldots\}$ refers to the statistical mean while Sp $\{\ldots\}$ indicates the trace with respect to spinor indices). If $f^{\alpha \beta}(\boldsymbol{p}, \boldsymbol{x}, t)$ is known one can calculate the mean of every one-particle dynamical quantity $\chi^{\alpha \beta}(\boldsymbol{x}, \boldsymbol{p})$,

$$
\bar{\chi}(\boldsymbol{x}, t)=\int \mathrm{d}^{3} p \operatorname{Sp}\{\chi(\boldsymbol{x}, \boldsymbol{p}) f(\boldsymbol{x}, \boldsymbol{p}, t)\},
$$

where eventually some divergence arising from radiative corrections of pure vacuum terms must be eliminated by the usual renormalization.

In contrast to the definition of the WIGNER function given above, BALEscu ${ }^{5}$ seems to consider $\psi(\boldsymbol{p})$ $\left(\psi^{+}(\boldsymbol{p})\right)$ as the destruction (creation) operator of a so called chargeon and to require normal ordering only with respect to $\psi^{+}(\boldsymbol{p}), \psi(\boldsymbol{p})$. Evidently the one-chargeon function would then be positive definite although it is supposed to describe the distribution of charges of both signs. This definition corresponds to a Hilbert space where in the sense of the Dirac hole theory no negative energy states are occupied. Since in this case transitions of positive energy electrons to negative energy states are possible, one has the paradoxial result that the system even if in thermal equilibrium is unstable at sufficiently high frequencies. We shall come back to this point in the next section.

Starting from the equation for the density operator, $\partial \varrho / \partial t=-i[H, \varrho(t)]$, and using the Hamiltonian (1) and the commutation relations for $\psi$ and $a$, we obtain the equations for the Fourier transform of the Wigner function, $f(\boldsymbol{p}, \boldsymbol{k})$, and the mean potential, $\left\langle A_{\mu}(\boldsymbol{k})\right\rangle(\langle\ldots\rangle$ stand for $\operatorname{Tr}\{\varrho \ldots\})$ :

$$
\begin{aligned}
& \frac{\partial}{\partial t} f^{\alpha \beta}(\boldsymbol{p}, \boldsymbol{k})+i(\boldsymbol{\alpha} \cdot(\boldsymbol{p}-\boldsymbol{k} / 2)+\beta m)^{\alpha \gamma} f^{\gamma \beta}(\boldsymbol{p}, \boldsymbol{k})-i f^{\alpha \gamma}(\boldsymbol{p}, \boldsymbol{k})(\boldsymbol{\alpha} \cdot(\boldsymbol{p}+\boldsymbol{k} / 2)+\beta m)^{\gamma \beta}= \\
= & i e \frac{1}{(2 \pi)^{3}} \sum_{\boldsymbol{k}^{\prime}}\left[\left(\alpha^{\mu}\right)^{\alpha \gamma}\left\langle: \psi^{+\beta}(\boldsymbol{p}+\boldsymbol{k} / 2) \psi^{\gamma}\left(\boldsymbol{p}-\boldsymbol{k} / 2-\boldsymbol{k}^{\prime}\right): A_{\mu}\left(\boldsymbol{k}^{\prime}\right)\right\rangle\right. \\
& \left.\quad-\left(\alpha^{\mu}\right)^{\gamma \beta}\left\langle: \psi^{+\gamma}\left(\boldsymbol{p}+\boldsymbol{k} / 2+\boldsymbol{k}^{\prime}\right) \psi^{\alpha}(\boldsymbol{p}-\boldsymbol{k} / 2): A_{\mu}\left(\boldsymbol{k}^{\prime}\right)\right\rangle\right] \\
& +i e \frac{1}{(2 \pi)^{3}}\left[\alpha^{\mu} \Lambda^{+}(\boldsymbol{p}+\boldsymbol{k} / 2)-\Lambda^{+}(\boldsymbol{p}-\boldsymbol{k} / 2) \alpha^{\mu}\right]^{\alpha \beta}\left\langle A_{\mu}(-\boldsymbol{k})\right\rangle, \\
& \left(\frac{\partial^{2}}{\partial t^{2}}+k^{2}\right)\left\langle A^{\mu}(\boldsymbol{k})\right\rangle=-4 \pi e \frac{(2 \pi)^{3}}{\Omega} \sum_{k} \operatorname{Sp}\left\{\alpha^{\mu} f(\boldsymbol{p},-\boldsymbol{k})\right\} \equiv 4 \pi j^{\mu}(\boldsymbol{k}) .
\end{aligned}
$$

These are the first equations of an infinite hierarchy involving more and more complex $\mathrm{W}_{\text {IGNER }}$ functions. To the lowest order (neglecting all correlations) ${ }^{7}$ the function $\left\langle: \psi^{+} \psi: A\right\rangle$ is just the product $f\langle A\rangle$, and (7) takes the form of a selfconsistent field equation:

$$
\begin{aligned}
& \frac{\partial}{\partial t} f(\boldsymbol{p}, \boldsymbol{k})+i(\boldsymbol{\alpha} \cdot(\boldsymbol{p}-\boldsymbol{k} / 2)+\beta m) f(\boldsymbol{p}, \boldsymbol{k})-i f(\boldsymbol{p}, \boldsymbol{k})(\boldsymbol{\alpha} \cdot(\boldsymbol{p}+\boldsymbol{k} / 2)+\beta m) \\
= & i e \sum_{\boldsymbol{k}^{\prime}}\left[\alpha^{\mu} f\left(\boldsymbol{p}-\boldsymbol{k}^{\prime} / 2, \boldsymbol{k}+\boldsymbol{k}^{\prime}\right)-f\left(\boldsymbol{p}+\boldsymbol{k}^{\prime} / 2, \boldsymbol{k}+\boldsymbol{k}^{\prime}\right) \alpha^{\mu}\right]\left\langle A_{\mu}\left(\boldsymbol{k}^{\prime}\right)\right\rangle \\
& +\frac{i e}{(2 \pi)^{3}}\left[\alpha^{\mu} \Lambda^{+}(\boldsymbol{p}+\boldsymbol{k} / 2)-\Lambda^{+}(\boldsymbol{p}-\boldsymbol{k} / 2) \alpha^{\mu}\right]\left\langle A_{\mu}(-\boldsymbol{k})\right\rangle .
\end{aligned}
$$

Eq. (9) has been given by Silin and Rukhadze ${ }^{8}$ without the last term. Though negligeable in the particular

7 Smallness parameter is $\alpha=e^{2} / \hbar c$ for the degenerate case, $e^{2} n^{1 / s} / k T=\alpha(\hbar / m c) n^{1 / s} \cdot m c^{2} / k T$ for the nondegenerate case.
8 V.P. Silin and A. A. Rukhadze, Soviet Phys.-JETP 11, 463 [1960]. 
case treated in their paper, this term (which arises from the definition of $f^{\alpha \beta}$ as a normal product) cannot in general be omitted. - Since we disregard external fields, $\left\langle A_{\mu}(0)\right\rangle=0$, and (9) reduces for a homogeneous distribution to

$$
\frac{\partial f(\boldsymbol{p})}{\partial t}+i(\boldsymbol{\alpha} \cdot \boldsymbol{p}+\beta m) f(\boldsymbol{p})-i f(\boldsymbol{p})(\boldsymbol{\alpha} \cdot \boldsymbol{p}+\beta m)=0 .
$$

For a homogeneous system one can always choose the states of the density matrix at the initial time such that $\Lambda^{+}(p) f(p, t=0) \Lambda^{-}(p)=0$. Then it follows from (10) that this quantity remains zero for every later time and $f(\boldsymbol{p})$ is constant in time. It is just the difference of the electron and positron distributions in momentum and spinor space, $f(\boldsymbol{p})=\left[\Lambda^{-}(\boldsymbol{p})+\Lambda^{+}(\boldsymbol{p})\right] f(\boldsymbol{p})=f^{-}(\boldsymbol{p})-f^{-}(\boldsymbol{p})$.

\section{The Dispersion Relation}

We now linearize (9) about a homogeneous unperturbed state and take the Fourier transform with respect to $t$ in the sense of switching on the interaction "adiabatically":

$$
\begin{gathered}
\omega f^{(1)}(\boldsymbol{p}, \boldsymbol{k}, \omega)+(\boldsymbol{\alpha} \cdot(\boldsymbol{p}-\boldsymbol{k} / 2)+\beta m) f^{(1)}(\boldsymbol{p}, \boldsymbol{k}, \omega)-f^{(+)}(\boldsymbol{p}, \boldsymbol{k}, \omega)(\boldsymbol{\alpha} \cdot(\boldsymbol{p}+\boldsymbol{k} / 2)+\beta m) \\
=e\left[\alpha^{\mu} f^{0}(\boldsymbol{p}+\boldsymbol{k} / 2)-f^{0}(\boldsymbol{p}-\boldsymbol{k} / 2) \alpha^{\mu}\right]\left\langle A_{\mu}(-\boldsymbol{k},-\omega)\right\rangle \\
+\frac{e}{(2 \pi)^{3}}\left[\alpha^{\mu} \Lambda^{+}(\boldsymbol{p}+\boldsymbol{k} / 2)-\Lambda^{+}\left(\boldsymbol{p}-\boldsymbol{k} / 2 \alpha^{\mu}\right]\left\langle A_{\mu}(-\boldsymbol{k},-\omega)\right\rangle .\right.
\end{gathered}
$$

From the solution of (11) we find the current:

$$
\begin{aligned}
j^{\mu}(\boldsymbol{k}, \omega) \equiv \Pi^{\mu \nu}(\boldsymbol{k}, \omega) A_{\nu}(\boldsymbol{k}, \omega)=-e \int \mathrm{d}^{3} p \operatorname{Sp}\left\{\alpha^{\mu} f^{(1)}(\boldsymbol{p},-\boldsymbol{k},-\omega)\right\} \\
\Pi^{\mu \nu}(\boldsymbol{k}, \omega)=e^{2} \int \mathrm{d}^{3} p \operatorname{Sp}\left\{\frac{\alpha^{\mu} \Lambda^{-}(\boldsymbol{p}+\boldsymbol{k} / 2) \alpha^{\nu} f_{0}^{-}(\boldsymbol{p}-\boldsymbol{k} / 2)-\alpha^{\mu} f_{0}^{-}(\boldsymbol{p}+\boldsymbol{k} / 2) \alpha^{\nu} \Lambda^{-}(\boldsymbol{p}-\boldsymbol{k} / 2)}{E_{\boldsymbol{p}+\boldsymbol{k} / 2}-E_{\boldsymbol{p}-\boldsymbol{k} / 2}+\omega+i \eta}\right. \\
+\frac{\alpha^{\mu} \Lambda^{+}(\boldsymbol{p}+\boldsymbol{k} / 2) \alpha^{\nu} f_{0}^{+}(\boldsymbol{p}-\boldsymbol{k} / 2)-\alpha^{\mu} f_{0}^{+}(\boldsymbol{p}+\boldsymbol{k} / 2) \alpha^{\nu} \Lambda^{+}(\boldsymbol{p}-\boldsymbol{k} / 2)}{E_{\boldsymbol{p}+\boldsymbol{k} / 2}-E_{\boldsymbol{p}-\boldsymbol{k} / 2}-\omega-i \eta} \\
+\frac{\alpha^{\mu} \Lambda^{+}(\boldsymbol{p}+\boldsymbol{k} / 2) \alpha^{\nu} f_{0}^{-}(\boldsymbol{p}-\boldsymbol{k} / 2)-\alpha^{\mu} f_{0}^{+}(\boldsymbol{p}+\boldsymbol{k} / 2) \alpha^{\nu} \Lambda^{+}(\boldsymbol{p}-\boldsymbol{k} / 2)}{E_{\boldsymbol{p}+\boldsymbol{k} / 2}+E_{\boldsymbol{p}-\boldsymbol{k} / 2}+\omega+i \eta} \\
\left.+\frac{\alpha^{\mu} \Lambda^{-}(\boldsymbol{p}+\boldsymbol{k} / 2) \alpha^{\nu} f_{0}^{+}(\boldsymbol{p}-\boldsymbol{k} / 2)-\alpha^{\mu} f_{0}^{-}(\boldsymbol{p}+\boldsymbol{k} / 2) \alpha^{\nu} \Lambda^{+}(\boldsymbol{p}-\boldsymbol{k} / 2)}{E_{\boldsymbol{p}+\boldsymbol{k} / 2}+E_{\boldsymbol{p}-\boldsymbol{k} / 2}-\omega-i \eta}\right\}+\delta \Pi_{0}^{\mu \nu}(\boldsymbol{k}, \omega) .
\end{aligned}
$$

The $\omega+i \eta(\eta=+0)$ in the denominators indicate the way of integration.

The last term $\delta \Pi_{0}{ }^{\mu \nu}$ arises from

$$
\begin{aligned}
\Pi_{0}{ }^{\mu \nu} \equiv & \frac{e^{2}}{(2 \pi)^{3}} \int \mathrm{d}^{3} p \\
& \cdot \operatorname{Sp}\left\{\frac{\alpha^{\mu} \Lambda^{+}(\boldsymbol{p}+\boldsymbol{k} / 2) \alpha^{\nu} \Lambda^{+}(\boldsymbol{p}-\boldsymbol{k} / 2)}{E_{\boldsymbol{p}+\boldsymbol{k} / 2}+E_{\boldsymbol{p}-\boldsymbol{k} / 2}-\omega-i \eta}\right. \\
& \left.+\frac{\alpha^{\mu} \Lambda^{-}(\boldsymbol{p}+\boldsymbol{k} / 2) \alpha^{\nu} \Lambda^{-}(\boldsymbol{p}-\boldsymbol{k} / 2)}{E_{\boldsymbol{p}+\boldsymbol{k} / 2}+E_{\boldsymbol{P}=\boldsymbol{k} / 2}+\omega+i \eta}\right\},
\end{aligned}
$$

which does not vanish in the limit $f^{ \pm}=0$, and diverges. For real $\omega, \Pi_{0}{ }^{\mu \nu}$ is just the vacuum polarisation part which is well known from quantum electrodynamics and which can be renormalized in the usual way. The only difference, which does not affect the renormalization, is the fact that (13) is not a causal but a retarded function $(\omega=\omega+i \eta$ instead of $\left.\omega^{2}=\omega^{2}-i \eta\right)$. This has the advantage, compared to the derivation of Tsytovich ${ }^{1}$, that $\Pi^{\mu \nu}$ has the correct imaginary part needed for the dispersion relation. The real part of the remaining expression $\delta \Pi_{0}{ }^{\mu \nu}$ is practically always negligeable; the imaginary part, however, is important, as it inhibits the system at thermal equilibrium from becoming unstable at very high frequences (pointed out by TsyTOVICH ${ }^{1}$, and independently by PFIRSCH).

Since the components $A^{\mu}$ are not independent because of the LoRENTz condition, the equation $\left(\omega^{2}-k^{2}\right) A^{\mu}+4 \pi \Pi^{\mu \nu} A_{\nu}=0$ does not lead directly to the dispersion relation $D(\omega, k)=0$. Using

$$
k^{i} A^{i}=\omega A^{0} \quad \text { and } \quad k^{i} \Pi^{i \mu}=\omega \Pi^{0 \mu},
$$

one finds the dispersion relation in the usual form:

$$
D(\omega, k) \equiv \operatorname{det}\left\|k^{i} k^{j}-\delta^{i j} k^{2}+\omega^{2} \varepsilon^{i j}\right\|=0
$$


where $\varepsilon^{i j}$ is the dielectric tensor given by the expression

$$
\varepsilon^{i j}=\delta^{i j}-\frac{4 \pi}{\omega^{2}} \Pi^{i j} .
$$

For thermal equilibrium we have

$$
f^{\mp}(\boldsymbol{p})=\frac{\Lambda^{\mp}(\boldsymbol{p})}{1+\exp \left\{\left(E_{\boldsymbol{P}} \mp \mu\right) / k T\right\}}
$$

and $\varepsilon^{i j}$ has the form

$$
\varepsilon^{i j}=\frac{k^{i} k j}{k^{2}} \varepsilon^{l}+\left(\delta^{i j}-\frac{k^{i} k j}{k^{2}}\right) \varepsilon^{t}
$$

for every isotropic system. For this case (15) reduces to the expressions given by Tsytovich ${ }^{1}$.

The author is very much indebted to Dr. D. PFIrSCH for his interest in this work and many valuable discussions.

\title{
Die Hochfrequenz-Resonanz-Sonde *
}

\author{
Günter Peter * * \\ Institut für Plasmaphysik, Garching bei München
}

(Z. Naturforschg. 22 a, 872-890 [1967] ; eingegangen am 9. Februar 1967)

\begin{abstract}
Dc and ac currents to a rf resonance probe were investigated experimentally in the frequency range from 1 to $100 \mathrm{Mc} / \mathrm{s}$ in a quiescent Cesium plasma. The measurements in the plasma are explained by calculations and measurements with a lumped-constant equivalent network ("plasma simulator").

It is shown that the ac current to the probe is strongly influenced by the capacitance of the cable leading to the probe. A device to compensate this cable-capacitance is developed and used. Furthermore, it is shown for the first time that the double (or devided) main resonance maximum, occurring frequently with dc and ac currents to the resonance probe, is generally due to the influence of a second reference electrode.

A simple and direct ("focus") method for very precise plasma density determination (from the intersection point of the rf-current-vs.-frequency curves with probe voltage as parameter) is developed and compared with ac transmission current and LANGMUIR probe measurements at plasma densities between $2 \times 10^{6}$ and $3 \times 10^{7} \mathrm{~cm}^{-3}$. Agreement is obtained between these three experimental methods within their margins of error. The comparison leads to a better understanding of the measured Langmuir characteristic and of the Cs-plasma of the "Alma I" device.
\end{abstract}

\section{I.1 Einführung}

Legt man an eine gewöhnliche Languurr-Sonde neben der Sondengleichspannung $U_{\text {so }}$ noch eine Hochfrequenzspannung $u_{\mathrm{HF}}$ an (Abb. 1), so fließen zusätzlich zum ursprünglichen Gleichstrom $I_{0}$ ein

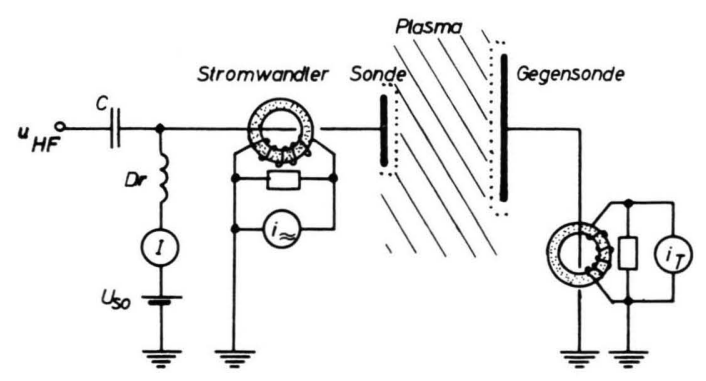

Abb. 1. Prinzipschaltung der HF-Sonde.

Transmissions-Wechselstrom $i_{\text {T }}$ und ein Zusatzgleichstrom $I=$ über die Sonde in das Plasma. Transmissions-Wechselstrom $i_{\mathrm{T}}$ und Zusatzgleichstrom $I_{=}$zei* Diese Arbeit wurde als Dissertation der Universität Mün-
chen angenommen (Juli 1966). gen, in Abhängigkeit von der Frequenz aufgetragen, ein Resonanzmaximum bei der Resonanzfrequenz $f_{\mathrm{r}}$, die in der Nähe, aber stets unterhalb der ElektronenPlasmafrequenz

$$
f_{\mathrm{p}}=\sqrt{\left(n_{\mathrm{e}} e^{2}\right) /\left(\pi m_{\mathrm{e}}\right)}=8,97 \cdot 10^{3} \sqrt{n_{\mathrm{e}}}\left(\mathrm{Hz} ; \mathrm{cm}^{-3}\right)
$$

liegt $\left(n_{\mathrm{e}}\right.$ bzw. $m_{\mathrm{e}}=$ Elektronendichte bzw. -masse, $e=$ positive Elementarladung). Der Zusatzgleichstrom $I_{=}$ist unterhalb der Resonanz (für $f \ll f_{\mathrm{r}}$ ) nahezu konstant und sinkt oberhalb der Resonanz rasch auf den Wert Null ab (vgl. Abb. 14 und 17 a). Der Transmissions-Wechselstrom $i_{\mathrm{T}}$ zur Sonde beginnt dagegen bei der Frequenz Null mit dem Wert Null, sinkt oberhalb der Resonanz nur auf ein Minimum ab und steigt bei noch höheren Frequenzen wieder an (vgl. Abb. 17 a).

Das Resonanzmaximum von Gleich- und Wechselstrom bei der Resonanzfrequenz $f_{r}$ ist auf eine Reihenresonanz zwischen dem sich unterhalb der

\footnotetext{
* Gegenwärtige Adresse: Laboratoire de Physique des Plasmas, École Royale Militatire, Brüssel 4, Belgien.
} 